\title{
X PREPARATION AND CHARACTERIZATION OF CARBON-BASED COMPOSITE NANOFIBERS FOR SUPERCAPACITOR
}

\author{
Dawei Gao', 2, Lili Wang', Chunxia Wang1', Yuping Chang'², Pibo Ma ${ }^{2 *}$ \\ ${ }^{1}$ College of Textiles and Clothes, Yancheng Institute of Technology, Yancheng 224051; \\ ${ }^{2}$ College of Textile and Clothing, Jiangnan University, Wuxi 214122 \\ E-mail:Gao D. gdw8668@163.com; Ma P. mapibo@jiangnan.edu.cn
}

\begin{abstract}
:
Polyacrylonitrile (PAN)/Co(OAc) _carbon nanotubes (CNTs) composite nanofibers were fabricated via electrospinning with $\mathrm{N}, \mathrm{N}$-dimethylformamide (DMF) as solvent, and by carbonization and activation of the above precursor nanofibers, porous carbon composite nanofibers were successfully obtained. Scanning electron microscope, $X$-ray diffraction, ASAP 2020, and Solartron 1470 were used to characterize the surface morphology, the phase composition, specific surface area, and electrochemical property of the nanofibers, respectively. The result showed that some of the fibers were broken after sintering, and the surface area and pore volume of the porous C/Cu/CNTs were $771 \mathrm{~m}^{2} / \mathrm{g}$ and $0.347 \mathrm{~cm}^{3} / \mathrm{g}$, respectively. The specific capacitance of the composite nanofibers reached up to $210 \mathrm{~F} / \mathrm{g}$ at the current density of $1.0 \mathrm{~A} / \mathrm{g}$. Its energy density and power density were $3.1 \mathrm{Wh} / \mathrm{Kg}$ and 2,337 W/Kg, respectively, at the current of 0.5 and $5 \mathrm{~mA}$.
\end{abstract}

\section{Keywords:}

electrospinning; carbon nanotube; nanofiber; electrochemical property; specific capacitance

\section{Introduction}

Chemical double-layer capacitor, also known as supercapacitor, is a new kind of energy storage device between static capacitor and traditional chemical power source. With the speedness of charging and discharging, high efficiency as well as long cycle life, it's widely used in portable electronic devices and cars [1-2] Porous carbon composites became one of the most commonly used electrode materials because of its good conductivity, electrochemical stability, and low cost [3]. Supercapacitor stores electricity by physically absorbing electrolyte ions to the surface of porous carbon electrode. Therefore, specific surface areas, pore size distributions, and electrical conductivity are key factors affecting the storage capacity of porous materials [4]. It's capacitance is not proportional to specific surface areas, so pore size distributions and electrical conductivity of the material need to be improved as well as specific surface areas of electrode materials in order to improve its electrochemical performances [5]. Porous materials are widely used in catalyst carriers, sensors, electronic materials, filter materials, and energy storage materials and ,therefore, have become a research hotspot.

Electrospinning is a technology to prepare top-down continuous micro- or nanofibers. Compared with traditional preparation methods of nanofibers such as chemical vapor deposition, laser vaporization, arc discharge, and template method, it's environmentally friendly and has advantages of simpler technique and higher efficiency. Meanwhile, electrospinning nanofibers have controllable diameters and bigger specific surface areas [6-7]. Carbon nanofibers treated in high temperatures have a better electrical conductivity and can be directly used as supercapacitor electrode materials without adding any conductive agent or adhesive [8-9].
Response surface methodology (RSM) is statistical methods using multiple quadratic regression equations to fit the function between factors and response values in order to solve multivariate problems [10-13], in which the method of second-order Box-Behnken designing (BBD) based on three levels needs less test data and has a higher efficiency, with experimental factors not in extreme levels simultaneously and all experimental points located in safe operation regions. So far, this method has been more widely used in optimizations of biomedicine [14], chemical synthesis procedure [15], food fermentation [16], and so on.

In recent years, using electrospinning polymer fibers, matrix of carbon fibers has gained more and more attention. Using polyacrylonitrile (PAN)/polyvinyl pyrrolidone (PVP)/Cu(OAc) and $N, N$-dimethylformamide (DMF) as precursor and solvent, respectively, porous carbon composite nanofibers were obtained at different ratios adopting electrospinning and hightemperature carbonization. Researches on its electrochemical capacitance performance were conducted, and the effects of different ratios on capacitance were systematically studied. As a result, its proportional parameters were optimized.

\section{Experiments}

\subsection{Materials and equipments}

PAN, molecular weight of 100,000 , the United States FaithGroup Co., Ltd; PVP, Sinopharm Chemical Reagent Co., Ltd; copper acetate $\left(\mathrm{Cu}(\mathrm{OAc})_{2}\right)$, Sinopharm Chemical Reagent Co., Ltd; DMF, Sinopharm Chemical Reagent Co., Ltd; carbon nanotubes (CNTs), Chengdu Institute of Organic Chemistry, 
Chinese Academy of Sciences; Homemade electrospinning equipments; S-4800 Scanning Electron Microscope; Rigaku Micromax-002 X-ray Diffractometer (R-AXIS IV++); ASAP 2020 Adsorption Instrument.

\subsection{Preparation of precursor solution}

A certain amount of CNTs were weighed and added to DMF solution and then vibrated for 8 hours in ultrasonic vibration generator till transparent homogeneous solution was formed. Then prepared PAN was added to aforementioned solution and stirred sufficiently for 2 hours. Lastly, $\mathrm{Cu}(\mathrm{OAc})_{2}$ was added to PAN/CNTs solution and stirred sequentially for 2 hours, after which PAN/PVP/ $\mathrm{Cu}(\mathrm{OAc}) 2$ composite nanofiber solvent was prepared as electrospinning solvent. The mass fraction of its solute was $10 \%$, and PAN occupied $90 \%$ of the content, while the mass fraction of CNTs was $0.5 \%$.

\subsection{Preparation of PAN/PVP/Cu(OAc) ${ }_{2}$ composite nanofibers}

Prepared spinning solution was poured into an injector with the capacity of $20 \mathrm{~mL}$ and the needle diameter of $0.5 \mathrm{~mm}$. During electrospinning procedure, a voltage of $15 \mathrm{kV}$ was applied to the needle, and the aluminum foil as a receiver was connected to earth. The needle and aluminum foil had a distance of 15 $\mathrm{cm}$, and the injection pump had a flux of $0.5 \mathrm{~mL} / \mathrm{h}$. Nanofibers collected from the aluminum foil was then prepared after drying. $\mathrm{PAN} / \mathrm{Cu}(\mathrm{OAc})_{2}$ composite nanofibers were also prepared for the purpose of comparison afterwards.

\subsection{Preparation of $\mathrm{C} / \mathrm{Cu} / \mathrm{CNTs}$ composite nanofibers}

Aforementioned precursor composite nanofibers were put into a box furnace, and detailed steps are as follows: first, heating the nanofibers to $250{ }^{\circ} \mathrm{C}$ at a rate of $2{ }^{\circ} \mathrm{C} / \mathrm{min}$ in air and keeping the temperature for 4 hours with an air flow rate of $20 \mathrm{PSI}$; second, under the protection of nitrogen, continuing heating up to $800^{\circ} \mathrm{C}$ at a rate of $5{ }^{\circ} \mathrm{C} / \mathrm{min}$ and keeping the temperature for 6 hours with a nitrogen flow of $20 \mathrm{PSI}$; Third, stopping pumping in nitrogen and switching to $\mathrm{CO}_{2}$ with a flow of $20 \mathrm{PSI}$, kept for 30 min under the temperature of $800{ }^{\circ} \mathrm{C}$; Fourth, switching $\mathrm{CO}_{2}$ to nitrogen with a flow of $20 \mathrm{PSI}$ till the temperature of the box furnace was cooled down to room temperature, after which the sample was prepared finally.

\section{$\underline{2.5}$ Test of electrochemical performance}

The test of electrochemical performance adopted a doubleelectrode system and was conducted using Solartron 1470 in Solartron Analytical, Houston, TX, with a electrode diameter of 1.25 $\mathrm{cm}$. $\mathrm{KOH}$ solvent with a mole fraction of $6.0 \mathrm{~mol} / \mathrm{L}$ was adopted.

\section{Results and discussion}

\section{$\underline{3.1 \text { Box-Behnken designing and analysis }}$}

Based on response values in Table 1 from the 17 experiments designed by Box and Behnken, the quadratic regression fitting was carried out and a quadratic equation with interaction items and quadratic terms was obtained. The main effects and interaction effects of various factors were analyzed, and the optimum value was obtained in a certain level. Significance tests of the regression equation were shown in Table 2.

Table 1. Results of experimental design by RSM

\begin{tabular}{|c|c|c|c|c|}
\hline \multirow{2}{*}{ Numbers } & \multicolumn{3}{|c|}{ Codes } & \multirow{2}{*}{$\begin{array}{c}C_{s p} \\
(F / g)\end{array}$} \\
\hline & $x$ & $\mathbf{Y}$ & $\mathbf{Z}$ & \\
\hline 1 & 0 & 0 & 0 & 99 \\
\hline 2 & 0 & 0 & 0 & 83 \\
\hline 3 & 1 & 0 & 1 & 54 \\
\hline 4 & 0 & 0 & 0 & 98 \\
\hline 5 & 0 & -1 & 1 & 50 \\
\hline 6 & 1 & 0 & -1 & 81 \\
\hline 7 & -1 & 0 & 1 & 57 \\
\hline 8 & 1 & -1 & 0 & 88 \\
\hline 9 & 0 & 1 & 1 & 55 \\
\hline 10 & -1 & 0 & -1 & 84 \\
\hline 11 & -1 & -1 & 0 & 93 \\
\hline 12 & -1 & 1 & 0 & 95 \\
\hline 13 & 0 & -1 & -1 & 57 \\
\hline 14 & 0 & 0 & 0 & 96 \\
\hline 15 & 0 & 0 & 0 & 98 \\
\hline 16 & 0 & 1 & -1 & 74 \\
\hline 17 & 1 & 1 & 0 & 86 \\
\hline
\end{tabular}

Table 2. Analysis of variance value of regression equation

\begin{tabular}{|c|c|c|c|c|}
\hline & $\begin{array}{c}\text { Coefficient } \\
\text { Estimates }\end{array}$ & $\begin{array}{c}\text { Standard } \\
\text { Deviation }\end{array}$ & $\begin{array}{c}\text { Values } \\
\text { of } \mathbf{F}\end{array}$ & $\begin{array}{c}\text { Values } \\
\text { of } \mathbf{P}\end{array}$ \\
\hline Intercepts & 94.80 & 3.14 & & \\
\hline $\mathrm{X}$ & -2.50 & 2.48 & 1.01 & 0.3476 \\
\hline $\mathrm{Y}$ & 2.75 & 2.48 & 1.23 & 0.3047 \\
\hline $\mathrm{Z}$ & -10.00 & 2.48 & 16.22 & 0.0050 \\
\hline $\mathrm{XY}$ & -1.00 & 3.51 & 0.081 & 0.7841 \\
\hline $\mathrm{XZ}$ & 0.000 & 3.51 & 0.000 & 1.0000 \\
\hline $\mathrm{YZ}$ & -3.00 & 3.51 & 0.73 & 0.4212 \\
\hline $\mathrm{X}^{2}$ & 2.85 & 3.42 & 0.69 & 0.4325 \\
\hline $\mathrm{Y}^{2}$ & -7.15 & 3.42 & 4,36 & 0.0751 \\
\hline
\end{tabular}

Quadratic multiple regression model obtained from regression analysis was as follows: $\mathrm{C}_{\mathrm{sp}}=94.8-2.50 \mathrm{X}+2.75 \mathrm{Y}-10.0 \mathrm{Z}-$ $1.0 X Y-3.0 Y Z+2.85 X^{2}-7.15 Y^{2}-28.65 Z^{2}$

It can be seen from Table 2 that the value of $R^{2}$ in regression equation is equal to 0.93 . And it showed that this equation has a better fitting degree and can be used for the prediction of 
capacitance. In the equation, $P$ values of both $Z$ and $Z^{2}$ are less than 0.05 , which means that the mass fraction of $\mathrm{Cu}(\mathrm{OAc})_{2}$ and its quadratic term have a significant influence on capacitance. The optimal ratio of PAN, PVP, and $\mathrm{Cu}(\mathrm{OAc})_{2}$ is 76:14:10. Simultaneously, the sample has a capacitance of $95.8 \mathrm{~F} / \mathrm{g}$ and has a difference ratio of $5.2 \%$ with predicted values, which shows that this model is affective in a certain range.

It can be directly known from Figure 1 that the content of PAN, PVP, and $\mathrm{Cu}(\mathrm{OAc})_{2}$ is not the higher and the better. The response curves of PVP and $\mathrm{Cu}(\mathrm{OAc})_{2}$ are a little steep, which means that interaction between PVP and $\mathrm{Cu}(\mathrm{OAc})_{2}$ is outstanding while interaction between PAN and PVP is unremarkable, and PVP has no interaction with $\mathrm{Cu}(\mathrm{OAc})_{2}$.
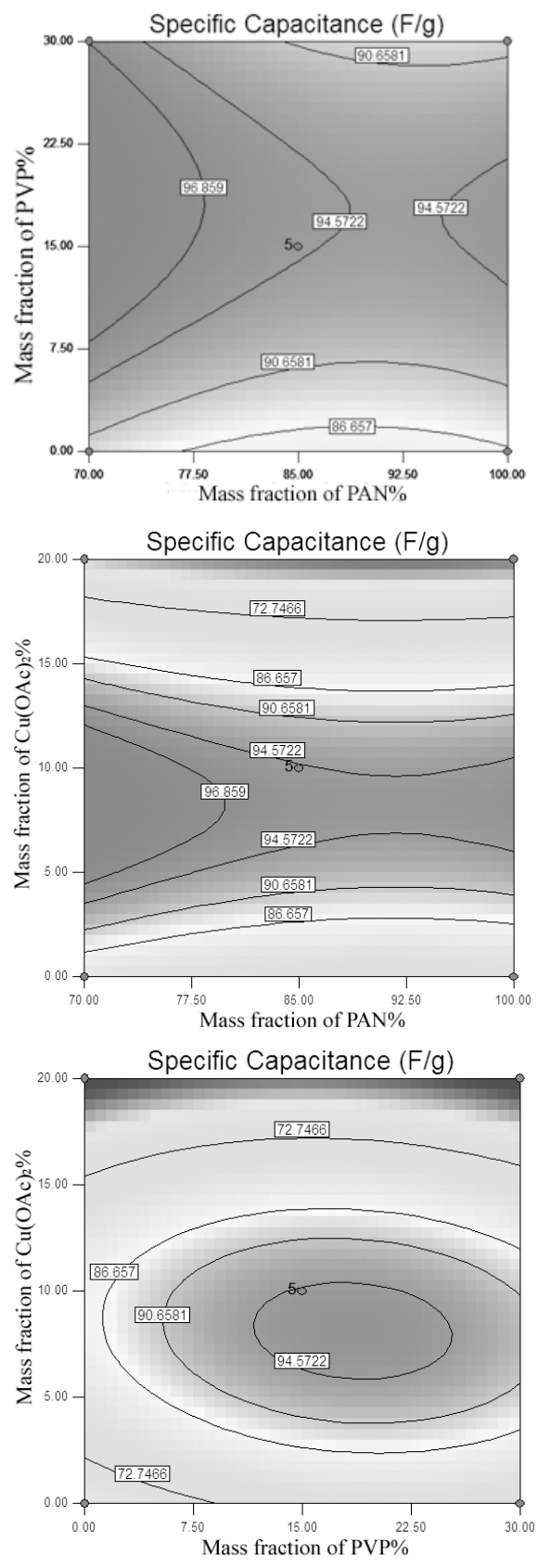

Figure 1. Contour plots showing the effects of the factors on response. (a) PAN and PVP concentration; (b) PAN and $\mathrm{Cu}(\mathrm{OAc})_{2}$ concentration; (c) PVP and $\mathrm{Cu}(\mathrm{OAc})_{2}$ concentration

\section{$\underline{3.2 \mathrm{X} \text { ray-diffraction analysis }}$}

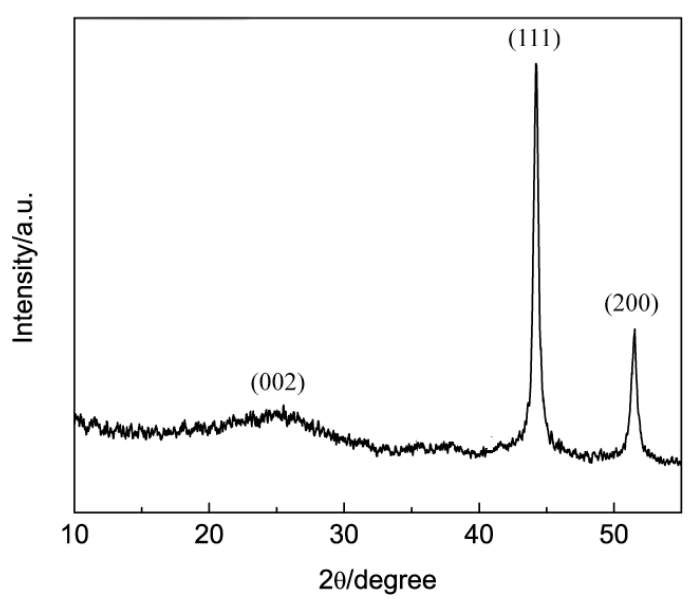

Figure 2. XRD patterns of carbon-based composite nanofibers

X-ray diffraction (XRD) patterns of $\mathrm{PAN} / \mathrm{Cu}(\mathrm{OAc})_{2} / \mathrm{CNTs}$ precursor composite nanofibers after high-temperature calcination are shown in Figure 2. It can be seen that there exists a wide peak at $2 \theta=25^{\circ}$ because of partial crystallization of carbon fibers, corresponding to crystal 002 (JCPDS 70-1478). Other two diffraction peaks are corresponded to pure copper 111 and crystal 200 (JCPDS 15-0806) with $2 \theta=44.2^{\circ}$ and $2 \theta=$ $51.5^{\circ}$, respectively. According to Scherrer equation, the average grain size that is perpendicular to the crystal 111 is $16.8 \mathrm{~nm}$.

\subsection{Scanning electron microscope analysis}

It can be observed from Figure 3(a) that surfaces of precursor $\mathrm{PAN} / \mathrm{Cu}(\mathrm{OAc})_{2} / \mathrm{CNTS}$ composite nanofibers are relatively smooth, while surfaces of partial fibers are still rugged, which is due to relatively even entanglement of PAN macromolecules dissolving in DMF. When spinning jet flow is speeding up in the electric field, PAN macromolecules stretch and align along the fiber's length. Molecular weight of $\mathrm{Cu}(\mathrm{OAc})_{2}$ in the surface of spinning jet flow is smaller, so that molecules move faster, while PAN molecules move slowly. With the volatilization of DMF, spots where molecules move fast dent while spots where molecules move slowly swell naturally.

As shown in Figure 3(b), diameters of $\mathrm{C} / \mathrm{Cu} / \mathrm{CNTs}$ composite nanofibers are thinner and brittle fracture occurs in partial fibers after high-temperature calcination. This is because $\mathrm{PAN}$ and $\mathrm{Cu}(\mathrm{OAc})_{2}$ discompose under high temperature and volatilize as gases during pre-oxidization and carbonization, and simultaneously, fiber weight decreases when carbon in composite nanofibers reacts with activator $\mathrm{CO}_{2}$ during activation stage. Besides, when calcined under high temperature, precursor composite nanofiber $\mathrm{PAN} / \mathrm{Cu}(\mathrm{OAc})_{2} / \mathrm{CNTs}$ brittly breaks at its weak links.

\section{$3.4 \quad \mathrm{~N}_{2}$ adsorption-desorption curves and pore size distribution plot}

It can be seen from Figure 4 that $\mathrm{N}_{2}$ isothermal absorptiondesorption curve of $\mathrm{C} / \mathrm{Cu} / \mathrm{CNTs}$ carbon composite nanofibers belongs to type I (IUPAC) and has a typical microporous 


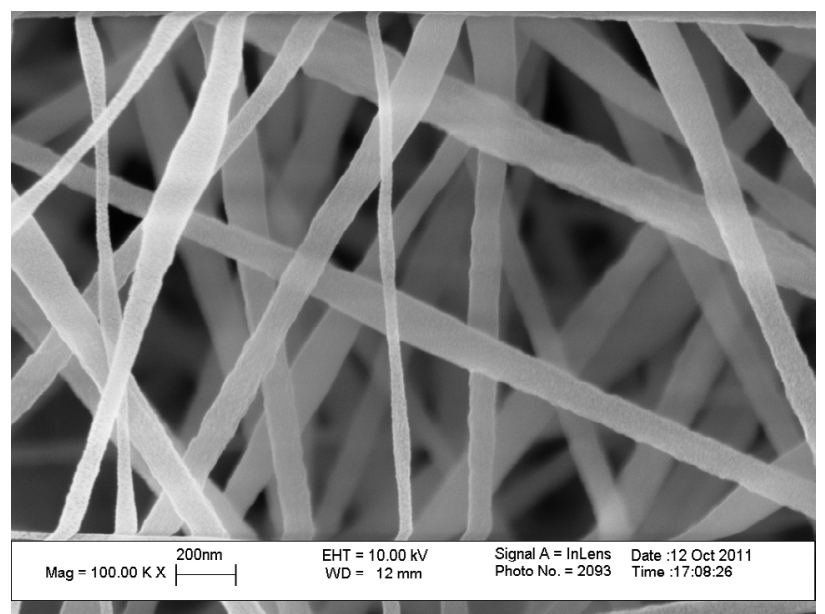

(a)

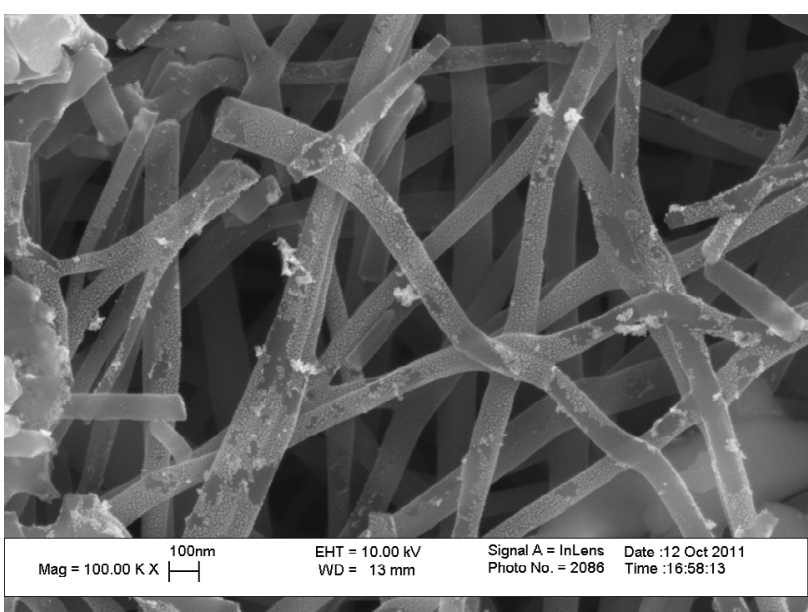

(b)

Figure 3. Scanning electron microscopic images of carbon composite nanofiber

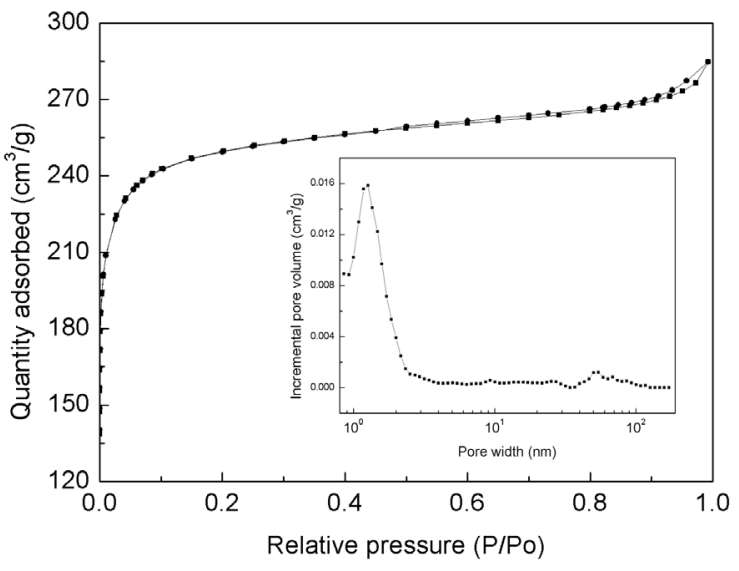

Figure 4. $\mathrm{N}_{2}$ adsorption-desorption isotherm and pore distribution plot (insert)

structure ${ }^{[20]}$. It can be observed from pore size distribution plot that pore sizes of $\mathrm{C} / \mathrm{Cu} / \mathrm{CNTs}$ composite nanofibers distribute narrowly, mainly between 0 and $2.0 \mathrm{~mm}$.

Data related to specific surface area and pore volume of samples are shown in Table 3. It shows that the specific surface area and pore volume of $\mathrm{C} / \mathrm{Cu} / \mathrm{CNTs}$ composite nanofiber are $771 \mathrm{~m}^{2} / \mathrm{g}$ and $0.347 \mathrm{~cm}^{3} / \mathrm{g}$, respectively, and increase by $53 \%$ and $35 \%$, respectively, compared with $\mathrm{C} / \mathrm{Cu}$ composite nanofibers. In addition, micropore, mesopore, and macropore coexist in nanofiber pores, in which micropore occupies the largest proportion in pores, that is, $79 \%$ and $92 \%$, respectively.

\subsection{Electrochemical performance analysis}

Cyclic voltammetry (CV) and galvanostatic charge-discharge were used to study electrochemical performance of $\mathrm{C} / \mathrm{Cu} / \mathrm{CNTs}$ composite nanofibers. Galvanostatic charge-discharge tests were carried out in the voltage range of $0-0.8 \mathrm{~V}$, with electric currents of $0.5,1.0,3.0$, and $5.0 \mathrm{~mA}$. Scanning speeds are set at 3,5 , and $10 \mathrm{mV} / \mathrm{s}$ when carrying out $\mathrm{CV}$ tests.

\subsubsection{Cyclic voltammetry tests}

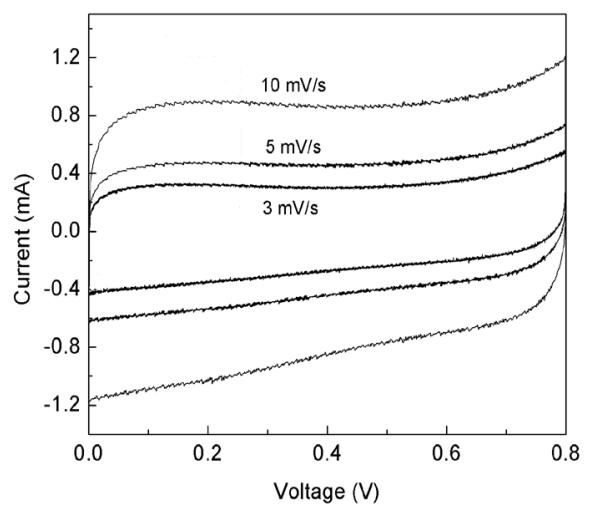

Figure 5. Cyclic voltammograms of carbon nanofibers

It can be seen from Figure 5 that cyclic voltammograms of samples approximate rectangles without reduction and oxidation peak, showing that this electrode material acts as

Table 3. Surface area and pore volume of carbon composite nanofiber

\begin{tabular}{|c|c|c|c|c|c|}
\hline \multirow{2}{*}{ Samples } & $\begin{array}{c}\text { Specific surface } \\
\text { areas } \\
\left(\mathbf{m}^{2} / \mathbf{g}\right)\end{array}$ & Micropore & Mesoporous & Macropore & Total volume \\
\cline { 3 - 6 } & 503 & 0.204 & 0.044 & 0.009 & 0.257 \\
\hline $\mathrm{C} / \mathrm{Cu}$ & 771 & 0.319 & 0.022 & 0.006 & 0.347 \\
\hline $\mathrm{C} / \mathrm{Cu} / \mathrm{CNTs}$ & & & \multicolumn{4}{|c|}{ Pore volumes $\left(\mathrm{cm}^{3} / \mathbf{g}\right)$} \\
\hline
\end{tabular}


capacitance almost totally because of its electrochemical double-layer capacitor. The shape of cyclic voltammetry curve had no obvious change with speeding up of scanning, indicating that composite nanofibers have a good capacity retention ratio and are suitable for electrode materials of supercapacitors.

\subsubsection{Galvanostatic charge-discharge tests}

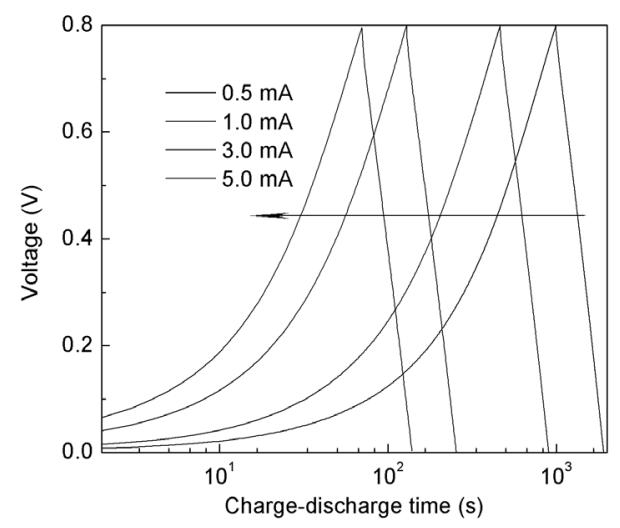

Figure 6. Charge-discharge curves of carbon nanofibers

As shown in Figure 6, charge-discharge time gets shorter with current increasing. This is because it takes long for electrolyte ions to get into fiber pores to form double-layer capacitance. When current density increases, it needs shorter time to form double layers and capacitance becomes smaller with electrolyte ions hindered. As charge-discharge current increases from 0.5 to $5.0 \mathrm{~mA}$, the discharge curve has no obvious potential drop in internal resistance, which means that this electrode material has good charge-discharge performances and has slight resistance to the spread of electrolyte ions.

\subsubsection{Effects of current density on specific capacitance}

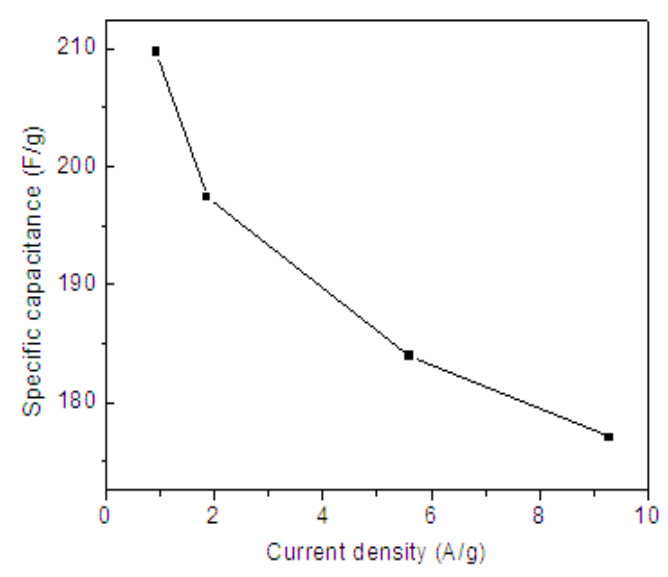

Figure 7. Specific capacitance as a function of current density

Figure 7 shows the specific capacitance curve as a function of current density. It can be seen that sample curves have good linear relationships under different current densities, which means that this electrode material has very good capacitance performance. When value of current density is $1.0 \mathrm{~A} / \mathrm{g}$, sample has a specific capacitance of $210 \mathrm{~F} / \mathrm{g}$, having increased about
$21 \%$ compared with $\mathrm{C} / \mathrm{Cu}$ composite nanofibers. C/Cu/CNTs composite nanofibers have larger specific surface areas and pore volumes to store more energies. Besides, the addition of CNTs forms a bridge structure between Cu particles, further improving its electrical conductivity as well as its electrochemical properties.

\subsubsection{Effects of electric current on power density and energy density}

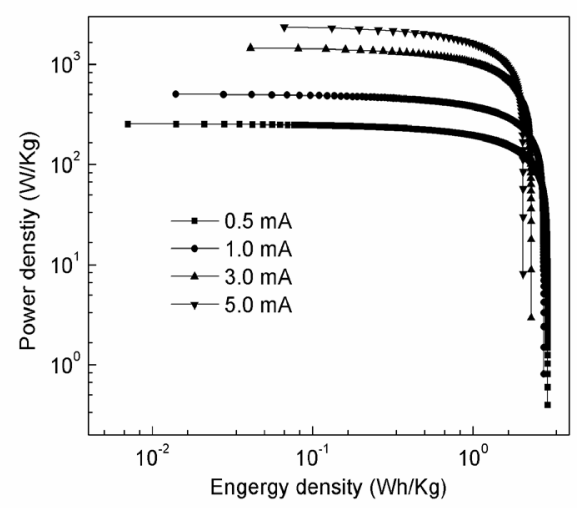

Figure 8. Ragone plots of carbon composite nanofibers

Figure 8 shows Ragone plots of carbon composite nanofibers. It can be seen that energy density of $\mathrm{C} / \mathrm{Cu} / \mathrm{CNTs}$ carbon composite nanofibers decreases as charge-discharge current increases. Its energy density and power density decreases from 3.1 to $2.7 \mathrm{Wh} / \mathrm{Kg}$ and increases from 256 to $2,337 \mathrm{~W} / \mathrm{Kg}$, respectively, when charge-discharge current increases from 0.5 to $5.0 \mathrm{~mA}$.

\section{Conclusion}

$\mathrm{PAN} / \mathrm{Cu}(\mathrm{OAc})_{2} / \mathrm{CNTS}$ composite nanofibers were prepared by electrospinning, and after treated under high temperatures, porous $\mathrm{C} / \mathrm{Cu} / \mathrm{CNTs}$ composite nanofibers were obtained. This kind of carbon composite nanofibers have higher specific surface areas, higher pore volumes, and narrower fiber pore size distributions. It can be used as electrode materials of supercapacitance without any conductive agent or adhesive agent. CNTs play a role of bridge between $\mathrm{Cu}$ particles and can improve its electrochemical properties by improving its electrical conductivity. Specific capacity of carbon composite nanofibers reaches $210 \mathrm{~F} / \mathrm{g}$ when the value of current density is $1.0 \mathrm{~A} / \mathrm{g}$.

Through response surface methodology, quadratic multiple regression models of PAN, PVP, and $\mathrm{Cu}(\mathrm{OAc})_{2}$ with different mass fractions to carbon composite nanofibers' capacitance were obtained. $\mathrm{Cu}(\mathrm{OAc})_{2}$ has the most significant effect, and secondly quadratic terms of $\mathrm{Cu}(\mathrm{OAc})_{2}$. Predicted values of the model have a good fitting with practical values. After treated under high temperatures, morphology of fibers become rough and fractures occur in partial fibers. XRD results show that components of optimized carbon composite nanofibers are $\mathrm{C} /$ $\mathrm{Cu} / \mathrm{CuO} / \mathrm{Cu}_{2} \mathrm{O}$. 


\section{Acknowledgments}

The authors would like to thank the Open Project Program of Key Laboratory of Eco-textiles, Ministry of Education, Jiangnan University (No. KLET1321), Yancheng Guidance Project of the Agricultural Science and Technology (YKN2014017)), the financial support from the Fundamental Research Funds for the Central Universities (No. JUSRP51625B), National Natural Science Funds of China (No. 11305138, 11302085), and the Innovation fund project of Cooperation among Industries, Universities \& Research Institutes of Jiangsu Province (No. BY2014023-34 and BY2014023-20).

\section{References}

[1] Huang X, Zeng Z, Fan Z, et al. Graphene-based electrodes, Advanced Materials, 2012, 24(45): 5979-6004.

[2] Zhu Y, Murali S, Stoller MD, et al. Carbon-Based Supercapacitors Produced by Activation of Graphene, Science, 2011, 332(6037): 1537-1541.

[3] Kim SY, Kim B-H, Yang KS, et al. Supercapacitive properties of porous carbon nanofibers via the electrospinning of metal alkoxide-graphene in polyacrylonitrile, Materials Letters, 2012, 87:157-161.

[4] Ma C, Song Y, Shi J, et al. Preparation and one-step activation of microporous carbon nanofibers for use as supercapacitor electrodes, Carbon, 2013, 51(1): 290-300.

[5] Hou Y, Chen L, Zhang L, et al. Ultrahigh capacitance of nanoporous metal enhanced conductive polymer pseudocapacitors, Journal of Power Sources, 2013, 225(0): 304-310.

[6] Duzyer S, Hockenberger $A$ and Zussman E. Characterization of solvent-spun polyester nanofibers, Journal of Applied Polymer Science, 2011, 120: 759-769.

[7] Zhang $\mathrm{ZY}$, $\mathrm{Li} \mathrm{XH}$, Wang $\mathrm{CH}$, et al. Polyacrylonitrile and Carbon Nanofibers with Controllable Nanoporous Structures by Electrospinning, Macromolecular Materials and Engineering, 2009, 294(10): 673-678.

[8] Zhi M, Manivannan A, Meng F, et al. Highly conductive electrospun carbon nanofiber $/ \mathrm{MnO}_{2}$ coaxial nano-cables for high energy and power density supercapacitors, Journal of Power Sources, 2012, 208:345-353.
[9] Zhou Z, Wu X-F and Fong H. Electrospun carbon nanofibers surface-grafted with vapor-grown carbon nanotubes as hierarchical electrodes for supercapacitors, Applied Physics Letters, 2012, 100(2):1-5.

[10] Box G.E.P. and Behnken D.W. Some new three level designs for the study of quantitative variables[J], Technometrics, 1960, 2(4): 455-475.

[11] Montgomery D.C. Design and Analysis of Experiments, 5th ed.[M], Wiley \& Sons: New York, 2002.

[12] Song Yeping, et al. Process optimization and prediction model of diameter for electrospun zein nanofibers[J], Journal of textile research, 2009, 30(7): 6-9.

[13] Zhang Lei-Yong, et al. Preparation and electrochemical properties of polyaniline/carbon nanofiber composite materials[J], acta physico-chimica sinica, 2010, 26 (12): 3181-3186.

[14] Sultana Shaheen, et al. Formulation development and optimization of alpha ketoglutarate nanoparticles for cyanide poisoning[J], Power technology, 2011, 211(1):1-9.

[17] Jyothi Alummoottil N., et al. Optimization of synthesis and characterization of cassava starch-graft-poly(acrylonitrile) using response surface methodology[J], Journal of applied polymer science, 2011, 122(3): 1546-1555.

[16] Jitrwung Rujira and Yargeau Viviane. Optimization of media composition for the production of biohydrogen from waste glycerol[J], International Journal of Hydrogen Energy, 2011, 36(16): 9602-9611.

[17] Matzui LY, Vovchenko LL, Kapitanchuk LM, et al. C-Co Nanocomposite Materials, Inorganic Materials, 2003, 39(11): 1147-1153.

[18] Wang L, Yu Y, Chen PC, et al. Electrospun carbon-cobalt composite nanofiber as an anode material for lithium ion batteries, Scripta Materialia, 2008, 58(5): 405-408.

[19] Tavanai $H$, Jalili $R$ and Morshed $M$. Effects of fiber diameter and $\mathrm{CO}_{2}$ activation temperature on the pore characteristics of polyacrylonitrile based activated carbon nanofibers, Surface and Interface Analysis, 2009, 41:814-819.

[20] Sing KSW, Everett DH, Haul RAW, et al. Reporting physisorption data for gas/solid systems with special reference to the determination of surface-area and porosity Pure and Applied Chemistry, 1985, 57(4): 603-619. 